\title{
Review on Stability of Thin-Walled Cylindrical Shells Subjected to Axial Compression
}

\author{
Haifeng Qian ${ }^{\mathrm{a}}$, Dengfeng Wang ${ }^{\mathrm{b}}$ * \\ School of Environment and Civil Engineering, Jiangnan University, Wuxi 214122, China \\ a1123408634@qq.com, bhappywdf@126.com
}

\begin{abstract}
Keywords:structural engineering; stability; review; cylindrical shells; axial compression; circumferential weld imperfection

Abstract. Thin-walled cylindrical shells under axial compression are commonly found in a wide range of civil engineering steel shell structures, including silos, towers, chimneys and tanks. Buckling of axially compressed cylindrical shells has been one of the most active areas of shell buckling research. The purpose of this paper is to review the globe study of the stability of thin-walled cylindrical shells subjected to axial compression, refine academic and engineering problems, and propose ideas for future research and development. Influence of initial geometric imperfection on stability of cylindrical shells subjected to axial compression is first introduced, followed by the influence of welding residual stress on the stability of cylindrical shells subjected to axial compression, as well as the influence of internal pressure on the stability of cylindrical shells subjected to axial compression. Meanwhile, suggestions for further research are proposed from the authors' point of view, offering reference for readers.
\end{abstract}

\section{Introduction}

Thin-walled cylindrical shells are widely used in chemical, electric power or food engineering, and it plays an increasingly important role in the national economic construction. At present, this kind of structure mostly adopts the simplified design method which is the main reason causing the failure of the thin-walled cylindrical shells, and become higher failure rate structure form [1]. Thin-walled cylindrical steel shell structures are often used as key facilities in the whole production system, once destroyed will cause the interruption of the production process, even lead to disaster. Therefore, it has important practical significance to study the bearing mechanism of thin-walled cylindrical steel shell structure. From the practical angle of design, this paper comprehensively summarizes the research results and the design criteria of the stability design of axial compression cylindrical shell. Influence of initial geometric imperfection on stability of cylindrical shells subjected to axial compression is first introduced, followed by the influence of welding residual stress on the stability of cylindrical shells subjected to axial compression, as well as the influence of internal pressure on the stability of cylindrical shells subjected to axial compression. The conclusion of this paper can be consulted by engineering design.

\section{Influence of initial geometric imperfection on stability of cylindrical shells subjected to axial compression}

Elastic classical buckling stress of ideal cylindrical shells subjected to uniform axial pressure by classical linear buckling analysis is shown in Eq. $1[2,3]$, the basic assumption is that perfect geometry, simply supported boundary conditions (one end constraint three translational degrees of freedom, one end constraint radial and circumferential displacement), the material is in elastic stage, and the shell is in the state of thin film stress. Thin film stress assumption means that the pre-buckling bending stress and deformation associated with boundary conditions were neglected in buckling analysis.

$$
\sigma_{\mathrm{cl}}=\frac{E}{\sqrt{3\left(1-v^{2}\right)}} \frac{t}{R} \approx 0.605 \frac{t}{R}
$$


In the equation, $E$ _ elastic modulus;

$$
\begin{aligned}
& \mathrm{V} \longrightarrow \text { poisson ratio (steel is } 0.3 \text { ); } \\
& R \longrightarrow \text { middle section radius of cylindrical shell; } \\
& t — \text { wallboard thickness of cylindrical shell. }
\end{aligned}
$$

Large-scale thin-walled cylindrical steel shell structure which widely used in civil engineering is usually used the following making method: first, roll smooth plate into surface shape and connect into a monolithic plate high cylindrical shell segment through the vertical weld, then, the shell segment is assembled into a complete cylindrical shell structure by means of circumferential welded seam, the construction process of the cylindrical steel shell structure is shown in Fig. 1. The measured results show that this kind cylindrical shell have the approximate axisymmetric initial geometrical imperfection at the place of circumferential weld seam[4], the shape of the imperfection is shown in Fig. 2. According to the measured data and the elastic bending theory of cylindrical shell, the various shape functions of the initial geometrical imperfections caused by the circumferential weld seam cooling shrinkage had been proposed by scholars worldwide[5], the shape functions of these imperfections are summarized in the literature[6], as shown in Fig. 3.

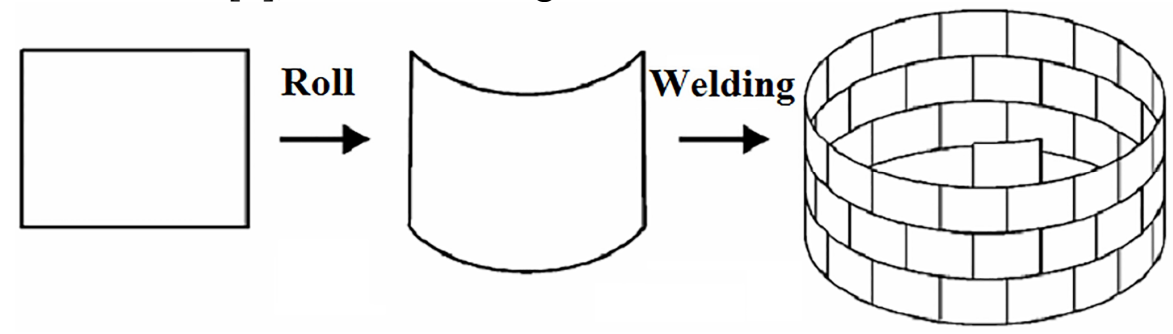

Fig. 1 Schematic diagram of the construction process of cylindrical shell structure

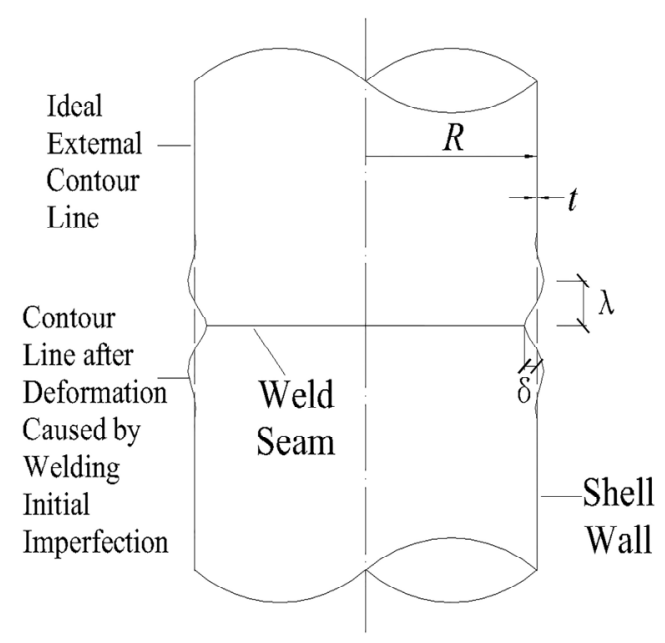

Fig. 2 Shape of the axisymmetric initial imperfection caused by welding

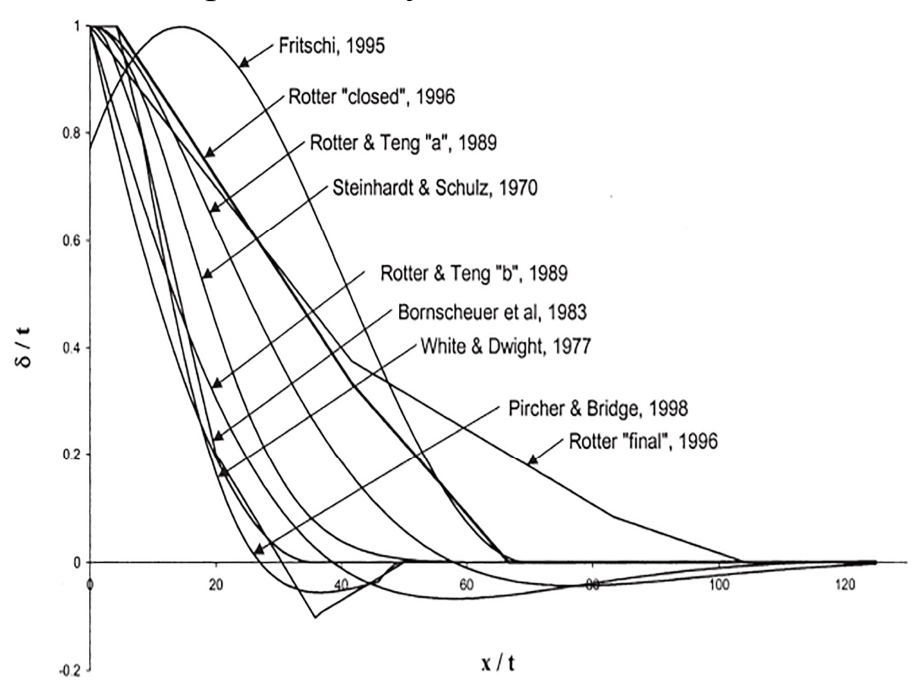

Fig. 3 Shape function of geometrical imperfection of different circumferential weld seams (quote from literature [6])

Literature [7] made statistical analysis on the measured imperfection of the test model, and made nonlinear finite element analysis on imperfection model based on probability, studied imperfection sensitivity. Literature [8] made the experimental and numerical study on compression behavior of nonslender cylindrical steel members with small and large-scale geometric imperfections. Literature [9] used the method of applying initial plate end displacement to study the initial geometric imperfection mode caused by the plate making assembly errors. 


\section{Influence of welding residual stress on the stability of cylindrical shells subjected to axial compression}

Large cylindrical shell is basically made of steel plate by roll and weld, the residual stress and the geometric imperfection will be produced in the production process. Because of the geometric imperfection have significantly effect on the bearing capacity of the shell, it is difficult to separate consider the effect of the residual stress and geometric imperfection in the test, only experimental study on the effect of residual stress is very difficult. Literature [10] shows that the residual stress can decrease the buckling stress of the cylindrical shell. However in this study, the assumed residual stress field compare with the no-load shell do not meet the equilibrium condition, and in the compared shells, the geometric imperfection amplitude whether the same is not explained, therefore, the reliability of the conclusion remains to be studied. In literature [11], the residual stress and initial geometric imperfections were simulated by applying a shrinkage strain(namely residual strain) field in the local area near the circumferential weld, the results show that the residual stress in the circumferential weld caused by welding shrinkage is favorable for the buckling strength of the cylindrical shell, and with the increase of the amplitude of the welding geometric imperfections, buckling load increase effect due to the consideration of the residual stress is obviously increased. Literature [6] and [12] are still used the method of the literature [11] which is by applying the residual strain to determine the uniform residual stress field in shell, the shell geometry changes caused by shrinkage strain are introduced as the initial geometric imperfection. The study proved that the residual stress has a certain increased effect on the buckling strength of cylindrical shell, in addition, the interaction between adjacent weld imperfections and the influence of boundary conditions were studied. The analysis shows that the interaction of adjacent weld can decrease the buckling strength if the strip height in adjacent weld is smaller; in addition, the buckling load obtained from the buckling mode on the symmetric of the middle height of the plate is maximum, the buckling load obtained from the antisymmetric buckling mode is the lowest, and the buckling load values obtained from the symmetric and antisymmetric modes are between the above two. The current design codes do not consider the effect of the residual stress, but the research shows that the effect of the residual stress is favorable, so neglect the effect of residual stress is on the safe side.

\section{Influence of internal pressure on the stability of cylindrical shells subjected to axial compression}

Literature [5] within the elastic range studied the influence of internal pressure on the stability of cylindrical shells subjected to axial compression. When cylindrical shell with initial geometric imperfections subjected to axial compression, the circumferential compression stress will be produced in the weld adjacent region, and its intensity is equivalent to the axial compression stress, the circumferential tensile stress caused by internal pressure of the cylindrical shell slow down the development of the circumferential compression stress, at the same time, the height range of shell under circumferential compression stress is reduced, therefore, the existence of internal pressure can improve the buckling stress of cylindrical shell, and the axial compression buckling stress increases with the increase of internal pressure. The results show that in the case of local concave imperfection in the place of weld seam, the internal pressure has the most improvement effect on the buckling strength, but the mutual influence of the adjacent two weld imperfections will decrease the increase of the buckling strength caused by the internal pressure.

The buckling strength of plastic buckling failure occurred in the bottom boundary vicinity of the cylindrical shell was studied by literature [13]. Stiffener cooperate with shell wall when it is set in internal pressure action area in the bottom of cylindrical shell, literature [14] put forward an effective cross section computational method which take the cooperation of the circumferential stiffener and shell wall into consideration, and put forward the suggestion of optimization design. Literature [15] studied the use of circumferential stiffener to improve the "elephant foot" buckling strength, the results show that the size of the stiffener is too large or small would both decrease the buckling strength, and put forward the suggestion of section optimization design. 


\section{Conclusions}

In this paper, the current situation of domestic and foreign research is summarized from three aspects of the influences of initial geometric imperfection, welding residual stress and internal pressure on stability of cylindrical shells subjected to axial compression; further research emphases and suggestions are provided for reference according to authors' consideration.

\section{References}

[1] TENG JinGuang, ZHAO Yang, Structural behavior and design of large steel silos, J. Journal of Civil Engineering.34 (2001) 46-55.

[2] Yamaki N, Elastic Stability of Circular Cylindrical Shells, Amsterdam, North Holland, 1984.

[3] HAN Qiang, HUANG XiaoQing, NING JianGuo, The Higher Shell Theory, Science Press, BeiJing, 2002, pp. 111-117.

[4] J.G.Teng, X.Lin, J.M.Rotter, X.L.Ding, Analysis of geometric imperfections in full-scale welded steel silos, J. Engineering Structures.27 (2005) 938-950.

[5] Teng J G, Rotter J M, Buckling of pressurized axisymmetrically imperfect cylinders under axial loads, J. Journal of Engineering Mechanics. 118 (1992) 229-247.

[6] Martin Pircher, Russell Bridge, The influence of circumferential weld-induced imperfections on the buckling of silos and tanks, J. Journal of Constructional Steel Research.57 (2001) 569-580.

[7] M.K.Chryssanthopoulos, M.J.Baker, P.J.Dowling, Statistical analysis of imperfections in stiffened cylinders, J. Journal of Structural Engineering.117 (1991) 1979-1997.

[8] Ilker Tutuncu, Thomas D.O'Rourke, Compression behavior of nonslender cylindrical steel members with small and large-scale geometric imperfections, J. Journal of Structural Engineering.132 (2006) 1234-1241.

[9] Holst J M F G, Rotter J M, Calladine C R, Characteristic geometric imperfection forms for cylinders derived from misfit calculations, Proceedings of International Conference on Carrying Capacity of Steel Shell Structures, Brno, 1997, pp. 333-339.

[10] Bornscheuer F W, Hafner L, Ramm E, Stability of a circular cylinder with a circumferential weld under axial loading, J. Stahlbau.52 (1983) 313-318.

[11]J.Mark F.G Holst, J.Michael Rotter, Chris R. Calladine, Imperfections and buckling in cylindrical shells with consistent residual stresses, J. Journal of Constructional Steel Research.54 (2000) 265-282.

[12] Martin Pircher, Russell Q.Bridge, Buckling of thin-walled silos and tanks under axial load-some new aspects, J. Journal of Structural Engineering. 127 (2001) 1129-1136.

[13]Rotter J M, Local inelastic collapse of pressurized thin cylindrical steel shells under axial compression, J. Journal of Structural Engineering.116 (1990) 1955-1970.

[14]J.F.Chen, J.M.Rotter, Effective cross sections of asymmetric rings on cylindrical shells, J. Journal of Structural Engineering. 124 (1998) 1074-1080.

[15]J.F.Chen, J.M.Rotter, J.G.Teng, Strengthening silos and tanks against elephant's foot buckling, in: Z.Y.Shen et.al, Advances in Steel Structures (Vol.1), Elsevier, Shanghai, 2005, pp. 459-466. 